\title{
Using Mobile Technology to Enhance Psychotherapy for Treatment of Schizophrenia: A Feasibility Study
}

\author{
Catherine Chambliss and April Kontostathis \\ Ursinus College, Department of Psychology \\ 646 Main Street \\ Collegeville, PA 19426-1000 \\ cchambliss@ursinus.edu
}

\begin{abstract}
Forty-two experienced clinicians who work with consumers diagnosed with schizophrenia were surveyed in order to assess the feasibility of using mobile applications as an adjunct to conventional treatment. Clinicians reported that over two-thirds of their consumers could safely make use of this technology. In addition, a majority of consumers were seen as likely to benefit from all but one of the fifteen functions performed by the investigational app (TherAPPist).
\end{abstract}

Keywords: Mobile application, Therapy, Schizophrenia.

(C) Copyright 2014 Authors - This is an Open Access article published under the Creative Commons Attribution License terms http://creativecommons.org/licenses/by/3.0). Unrestricted use, distribution, and reproduction in any medium are permitted, provided the original work is properly cited.

\section{Introduction}

Technology provides increasingly powerful tools for communication and accessing information. Psychotherapy applications tailored to meet the needs of those diagnosed with schizophrenia could provide individualized, immediate, and cost-effective interventions, using convenient devices that are steadily becoming more affordable. These mobile applications, such as TherAPPist, represent a selfregulation tool that may be a valuable adjunct to traditional treatment and asset to recovery.

The investigational TherAPPist app was developed by the authors to enhance the effectiveness of traditional psychopharmacologic and psychotherapeutic interventions. Released in April 2013, it currently provides the user with an easily accessible relaxation routine, positive refocusing stimuli, images of supporters with affirming messages, and emergency contacts. Its development continues, with plans to allow customization of images and audio, as well as tracking of target behaviors and affective outcomes.

The immediate access to therapeutic refresher messages afforded by the TherAPPist application was intended to improve generalization of therapeutic learning. This app offers immediate redirection and distraction, and provides individualized recovery cues. Therapists can't be there all the time, but therAPPist can be! This customizable app can put images and voices of supporters at the user's fingertips, 24/7.

Use of mobile applications presents a valuable opportunity to improve outcomes for individuals diagnosed with schizophrenia spectrum disorders by supplying timely therapeutic redirection, distraction, and coping tactics in highly challenging, provocative situations. A customizable mobile phone app can contribute to the development of confidence by providing images, video, sound and text that are meaningful for the individual user. The user's involvement in the customization process can foster a sense of ownership that may reduce resistance to following therapeutic suggestions.

An effective mobile device application can not only deter disruptive outbursts, but also improve treatment overall. Progress can be conveniently monitored; troubleshooting can be improved with more reliable data about triggering situations. In addition, immediate, personalized reinforcement can be delivered to encourage progress. This may help address some of the negative symptoms characterizing schizophrenia, 
including apathy, amotivation, and anhedonia [1]. In addition, the app can expand to reflect new learning, and serve as a visible product of recovery success. The objective is not to eliminate the need for professional support, but instead to provide real-time support so that consumers can function more effectively, especially in stressful or emotionally charged situations.

An app can also assist individuals in the day to day management of their illness by providing tools to track the correlation between mood and habits (such as taking medication), and offering an opportunity to journal in stressful situations. Furthermore, the information tracked by the mobile device app can be reviewed as part of the conventional therapy process, providing valuable data to both the consumer and the mental health provider.Learning to use the app also builds users' familiarity with current technology, helps to update their skills, and provides a "normalizing" experience.

\section{Development of TherAPPist}

This innovative app was designed to provide support on a variety of levels, everything from intervention in a high stress or emergency situation, to capabilities for tracking their treatment progress through the use of daily logs and goal tracking. The most significant feature of the app is the ability for it to be personalized and also its intended use as an integrated part of traditional therapy. When necessary, a therapist could help a client to tailor the app by discussing common stressors, as well as helpful images, voice, or text that may produce calming in a stressful situation. For example, one client may prefer to hear a voice clip of his therapist reminding him of the appropriate reactions. Another may prefer to see an image of her pet or a list of her goals. The app will be flexible so that multiple interventions can be tried until a successful one is found. Additionally, users can be encouraged to use the app on a daily basis to track situations and their associated emotions. This information will then be readily available for discussion at the next session.

Generally a chronic, lifelong disorder that frequently impairs functioning from early adulthood, schizophrenia continues to be quite challenging to treat.
Because medication compliance figures prominently in relapse prevention, one significant program feature will be the automation of reminders for medication compliance, and also tracking features that will show the correlation between positive habits (taking medicine, getting enough sleep), and outcomes (measured by mood, stress, etc.). Improvement in the area of conscientious management of sometimes complex medication regimes should contribute to recovery success.

\subsection{Overview of the Primary Features of TherAPPist}

The TherAPPist application is designed to provide features that will be particularly helpful to individuals with schizophrenia spectrum disorders. The application is intended to help consumers:

- Maintain self-control and manage anxiety, anger, and depression, as well as enhance social skills, decision making skills, and confidence.

- Reinforce the correlation between positive behaviors and outcomes, using simple, nonintrusive tracking mechanisms.

- Be completely customizable so that the user can select the images and media files that are most useful to him/her.

- Provide a valuable source of data which the consumer and his/her therapist can use to evaluate treatment effectiveness.

When a client first starts using TherAPPist as part of his treatment program, the therapist works with him to customize the app. Whenever practical, default options will automatically be selected to make the customization faster and smoother. After the initial setup, the user will have the ability to change their selections through a series of options menus.

The Relaxation routine is an audio relaxation induction. Soothing Scenes provides a selection of positive images to invite refocusing and return to a happier baseline. Supportive smiles presents a series of friendly images and phrases. Emergency numbers is a list of the most important contacts for easy access. The Relaxation Routine option is in the foremost spot, making it readily available in high-stress situations. 

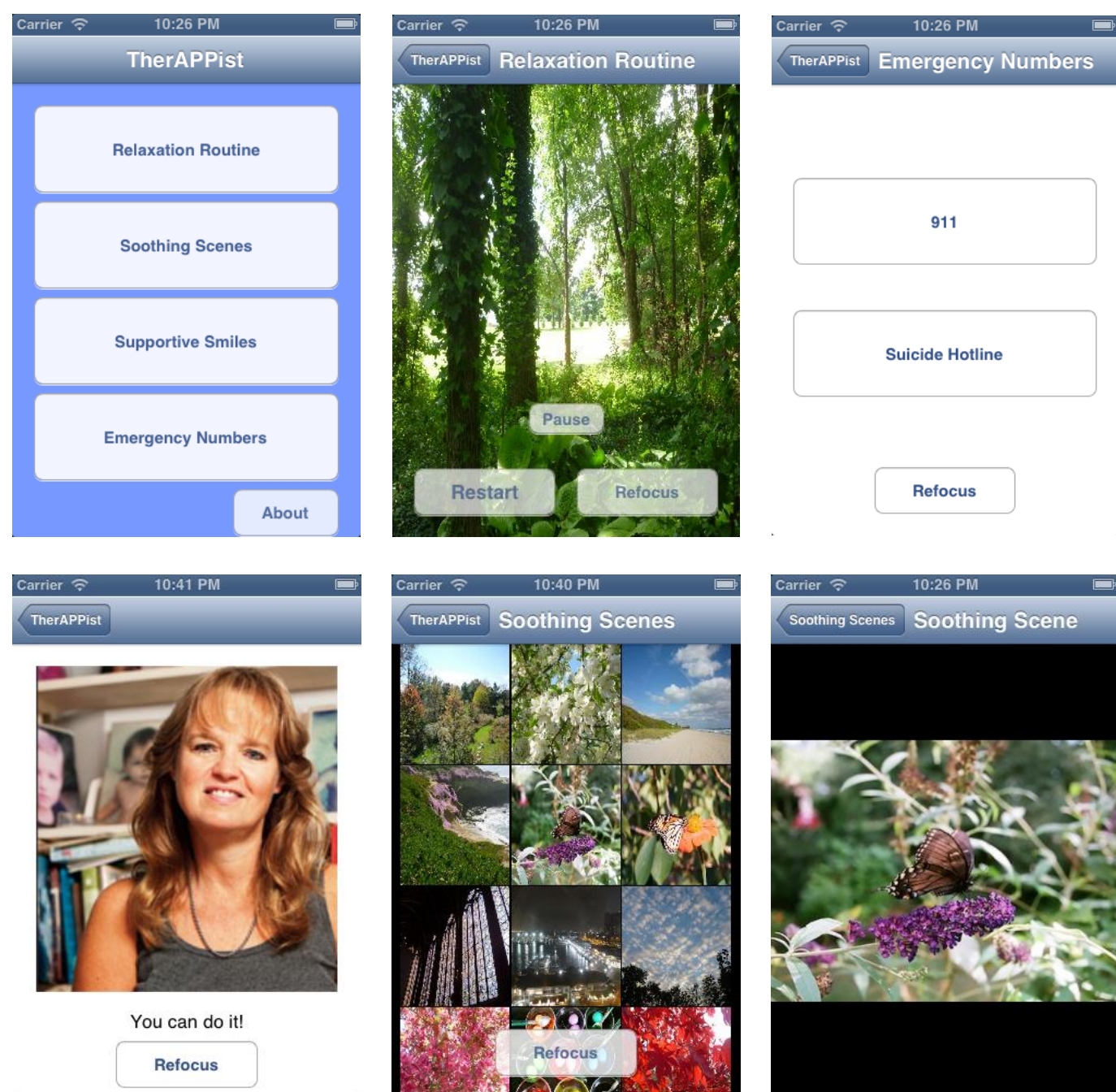

\begin{tabular}{c} 
You can do it! \\
Refocus \\
\hline
\end{tabular}

Figure 1. Several images from version 1 of TherAPPist app.

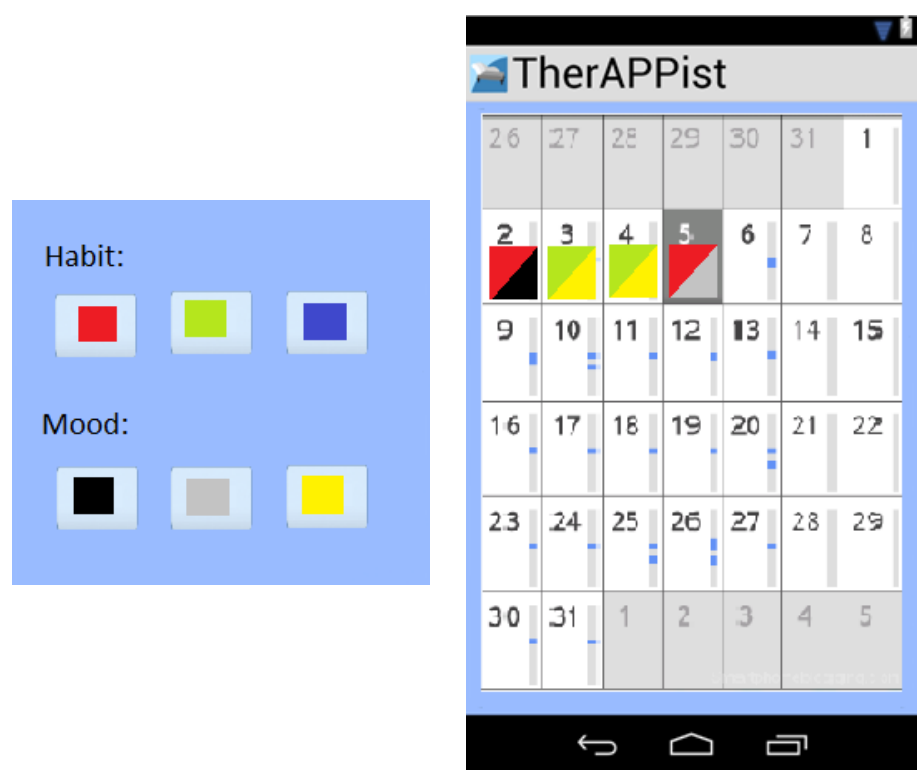

Figure 2. The Planned Tracker Feature. 
Figure 2 shows an early mockup of the habit/mood tracking feature of the app. The user will be prompted with reminder messages (outside the app) at a preconfigured time each day, and will be asked to use a simple 3-color scale to identify both the adherence to the habit being tracked (sleep, eating right, taking medicine), and also the mood. The user can then review the month (or partial month) with the therapist, so that visual patterns can be identified. For example, in Figure 2 , a "red/black" day on the $2^{\text {nd }}$ shows clearly that nonadherence to habit can result in negative effects on mood, whiles days 3 and 4 show that better (still not perfect) adherence to habit can greatly improve mood. The customization features will allow multiple habits and outcomes to be easily be tracked and shown on multiple calendars.

A medication tracker (to prompt the user when medicine should be taken) and a journaling feature are also planned. Additional life-management and redirection features will be added as time permits. All of these features will provide valuable data to both the individual and the therapist. Participants in the clinical trials will provide valuable feedback to determine the priority order for adding supplementary features.

There are four operating systems that are commonly in use for mobile devices: iOS (for iPhone and iPad), Android, Windows Phone 7, and BlackBerry OS. Of these systems, iOS and Android are the most commonly used, with $53 \%$ and $28 \%$ of the market, respectively [26]; therefore, we initially developed apps for these two operating systems. Version 1.0 (shown in Figure 1) is already available for download for users of both Android and iOS devices.

Preliminary informal surveys of consumers affected by schizophrenic spectrum disorder issues leave us encouraged that this app could be very useful. When groups of consumers have been asked about whether such an app would be helpful, they have expressed near universal enthusiasm. Many have stated that it would be "cool" to pull out a phone in times of stress. Most maintain that rapid access to helpful statements, images, and sounds would assist them in regulating emotion. They liked the notion of being able to choose from options on a menu, in order to tailor the helping process to their own tastes and preferences. Many liked the idea of being able to install their favorite affirming self-statements. Several cheered the chance to track their progress. They all seemed optimistic about the potential of such a device to increase their happiness; most felt it might help to defuse some tough situations.

An informal query of clinicians revealed that most agreed that this app has the potential to benefit many of their consumers. Reassuringly, most of the therapists queried indicated that they expected only a minority of their consumers to have difficulty mastering use of a simplified app, and only a minority of consumers (12.5 $\%$ ) was judged to be potentially unsafe with such a device. It was also heartening to find that a majority of the therapists expressed a willingness to try to incorporate use of the app in their ongoing work with some consumers. All viewed it as a useful adjunct to treatment, rather than as a competitor. Finally, some mentioned that the app's providing of guidance in a more impersonal way might reduce reactance and oppositional responding. Instead of feeling "nagged" by family, friends, and therapists, the app could provide an empowering means for consumers to receive timely recovery and wellness information.

The current study assessed the feasibility of using this mobile device with consumers diagnosed with schizophrenic spectrum disorders by surveying experienced clinicians. Respondents indicated the prevalence among their consumers of various problems addressed byTherAPPist. They also estimated the percentage of their consumers, both in inpatient residential care and in community residences, who could safety make use of a mobile phone.

\section{Related Work}

Although many forms of treatment are helpful, and medication generally reduces symptoms, relapse is an ongoing problem for many consumers with schizophrenia. Medication non-adherence figures prominently in this, as do other difficulties related to structuring time and activities on one's own. Problems with maintaining social contact and support also contribute to relapse. Research has shown that developing a relapse prevention plan and teaching strategies for coping with persistent symptoms measurably improve outcomes [2]. Medication adherence and persistence in schizophrenia is alarmingly low. According to Lieberman, Stroup, and McEvoy [3] between $64-82 \%$ discontinued initial medication within 18 months. Unfortunately, even brief medication lapses can have serious and costly consequences. For example, Weiden [4] found a twofold increased risk of hospitalization following nonadherance as brief as 10 days. 
Since remembering to deploy various lessons learned in therapy remains a challenge for many consumers with schizophrenia and schizoaffective disorder, the application's main value may lie in its capacity to prompt, remind, and enhance generalization. However, in addition to automating medication reminders and enabling therapists to detect non-adherence early on, and providing immediate relaxation and refocusing aides, TherAPPist also bridges the discharge support gapby augmenting the social support systemin a cost-effective way. Often individuals who do beautifully in settings where others are always available to prompt responses and offer reminders, or to offer redirection and distraction, deteriorate after moving to less structured settings with less ongoing contact with support staff.

Treatment of schizophrenia remains a challenge, despite dramatic improvements in psychopharmacotherapy and verbal psychotherapy. Increasingly, psychotherapy researchers have sought to identify best clinical practices through empirical investigations, in order to delineate evidence-based treatments for persons with these illnesses [5]. The randomized, controlled trial paradigm has yielded confidence in approaches such as The Illness Management and Recovery Program [6], Social Skills Training [7], and Relapse Prevention Training [8]. A meta-analytic evaluation of 27 social skills training (SST) studies involving schizophrenic persons revealed that SST dramatically improved patients' behavior in social situations, assertiveness and self-confidence, and hospital-discharge rate. Social Skills Training also helped to lower relapse rates [7]. This study was less definitive about the successful generalization of skills developed via social skills training.

In the treatment of schizophrenia, generalization of therapeutic learning is often limited. For example, many addressing self-control problems respond well during treatment sessions, but fail to deploy their new coping strategies in their daily lives. Shohamy, Mihalakos, Chin, Thomas, Wagner, and Tamminga [9] found individuals with schizophrenia to be selectively impaired in their ability to generalize knowledge, despite having intact learning and memory accuracy. Efforts to enhance generalization, including role play, are somewhat successful, but often work only in less emotionally charged situations [10]. When situational provocation is strong, and anger or fear levels are high, all too often the client resorts to their earlier (often self-destructive or self-defeating) mode of responding. Learning how to slow down and reflect before acting is extremely difficult for some clients. Use of simple, memorable strategies (e.g., Stop-Think-Relax; [11]) can help clients to give themselves time to process information carefully before running the risk of overreacting. However, again and again, many clients unfortunately forget to use even these easy tactics.

Enhancing generalization by augmenting conventional social skills training with specially designed mobile applications that offer immediate "refresher" experiences mayenhance treatment. Consistent with this, Kopelowicz, Liberman, and Zarate [12] argued that while beneficial, social skills training was not meant to function alone. They endorsed the possible incorporation of outside tactics into the actual methods used for social skills training, because they saw this as necessary for successful treatment.

A variety of other psychoeducational interventions have been shown to increase consumers' basic knowledge about their psychiatric illness, and relapse prevention programs also have been found to be helpful $[8,13]$. Although several psychosocial interventions are associated with improved functioning among those with schizophrenia, unfortunately these evidence-based practices have not been routinely available to many consumers who might profit from them [14]. Using mobile technology to make these useful practices more widely available could also benefit many consumers.

Many patients with schizophrenia experience a relapse after hospital discharge [15], often due to problems handling the lack of structure and support [16]. The TherAPPist app was designed to provide a mechanism for bridging this support gap for those in recovery, and can provide valuable data about the individual's use of various tactics that they have learned in therapy.This data can then be used by the individual and his/her therapist to alter treatment strategies appropriately.

Although The Illness Management and Recovery Program has been found to yield significant improvement in overall outcome, knowledge about illness, and progress in achieving personal goals, it has proven less successful for producing reliable gains on measures of social support, despite its aim to help clients improve their symptom management and social $[6,17]$. Hasson-Ohayon, Roe, and Kravetz [6] suggest that because applying coping techniquesin challenging situations and establishing and maintaining relationships and are more complex and demanding than internalizing information about illness and 
identifying personal goals, additional methods of assistance may be needed to achieve these important recovery objectives. The TherAPPist app may enable greater progress in these areas.

This app may be especially helpful for those who withdraw and isolate during times of stress. Unfortunately, this is quite common, because mistrust among these consumers is pervasive and often complicates the treatment process. Frequently, a history of interpersonal struggles marked by criticism, rejection, confusion, miscommunication, and mutual fear has left consumers suspicious of others. Some have particular difficulty reaching out to others when they most need support and redirection, at times of high negative emotional intensity. Many prefer at those challenging times to withdraw socially, but unfortunately lack reliably effective self-soothing strategies. Individuals with schizophrenia often cope with stress in a relatively ineffectual manner, often leading to poor outcomes [18]. Some maintain that the dangerously high smoking rates in this population $(90 \%)$ are due to attempts of individuals to selfmedicate; nicotine's up-regulation of GABA reduces the overstimulation, and many schizophrenics are unable to manage with other self-calming strategies. TherAPPistis intended to simplify the coping process by permitting consumers to provide themselves with calming cues and refocusing prompts. Hopefully, these rapid reminders will help them to manage negative feelings more constructively and reorient their attention positively.

At times of heightened stress, many consumers cannot generate constructive behavioral or cognitive options on their own. Because a support person is not always available at these moments to redirect attention and cue optimal responses, problem behaviors arise and crises escalate unnecessarily. Once skilled in activating this app, the consumer will be able to more self-sufficiently manage challenges. This should enhance perceived self-efficacy, further contributing to resilience and recovery. Believing that you can handle tough moments independently can be very empowering.

Recently much attention has focused on development of early interventions that may reduce damage resulting from repeated episodes of psychoses. An example of this approach, Recovery After an Initial Schizophrenia Episode (RAISE) is aimed at applying a multimodal aggressive response to the first episode of psychosis. This project seeks to improve the prognosis of schizophrenia through systematic interventions during the earliest stages of schizophrenic illness. RAISE is designed to reduce the long-term disability commonly associated with this illness by fostering achievement of recovery goals of independence and productivity. This should reduce the costs associated with long-term care. Mobile applications could assist programs such as RAISE in reducing the risk of longterm disability by fostering early medication adherence and other wellness objectives, including those related to sleep hygiene, nutrition, exercise, and maintenance of social support.

\section{Other Health Mobile Applications}

Use of technology, particularly mobile technology, to assist with managing illness and maintaining healthy habits is increasingly popular. There are a variety of apps that are designed to help motivate and track healthy habits for those who are trying to lose weight, for example. Two such apps, Livestrong'sMyPlate and Arawella Corporations' Calorie Counter, provide functions for tracking food, nutrition and calories, as well as activity, on a daily basis. They also provide long term progress charts and graphs. Millions of mobile device users have downloaded and use these apps regularly.

Moreover, there is growing evidence that these apps are effective. An evaluation of Aurora, a mobile phone based app for emotion recording and sharing, showed that the app encourages people to be more aware of their emotions and leads them to engage in socially supportive behavior [19]. A similar study by Deleeuw et. al [20] comes to the same conclusion. Additional evidence suggests that apps which run on mobile phones can be effective tools for the management of chronic diseases such as diabetes, hypertension, and asthma [21, 22, 23]. The National Library of Medicine (NLM) maintains a "Gallery of Mobile Apps and Sites" which features apps for tracking medications (MyMedList), accessing consumer information about drugs (DailyMed) as well as general research (access to PubMed for Handhelds), and specific health related information, such as LactMed for nursing mothers, and AIDSinfo mobile for AIDS patients and their doctors (http://www.nlm.nih.gov/mobile/). PmEB, a mobile device app for monitoring caloric balance in obese children and adults, was used to develop a heightened sense of self-awareness and promote self-monitoring [24]. 
According to a recent PEW survey, $83 \%$ of American Adults own some kind of cell phone, and 35\% own a smart phone of some kind. In the previous 30 days, $51 \%$ of adult cell phone owners had used their phones at least once to get information that they needed right away, $40 \%$ had used the phone in an emergency situation, and $42 \%$ had used the phone to stave off boredom [25].

\section{Method}

Forty-two psychologists, social workers, and therapeutic support aides(18 [43\%] male, 24 [57\%] female)who regularly provide services to consumers diagnosed with schizophrenia,as well as othersevere mental illnesses,served as participants in the current investigation (mean age: 45.67 years; mean years of clinical experience: 18.30 years). Surveys were administered to professional continuing education program attendees, prior to a discussion about the potential advantages and disadvantages of using mobile application technology as an adjunct to treatment. The survey assessed the clinician's years of experience working with MH/ID consumers, as well as the therapist's age and sex. Respondents were asked how many of their current consumers benefit from 15 functions scheduled to be offered by the investigational mobile application, including the following: Help using skills learned in therapy outside of treatment; redirection when upset; relaxation induction when upset; reminders about their strengths; reminders about supportive others; positive activities when bored; listening to music when upset; refocusing when upset; reminders to take medication; journaling; monitoring their goal behaviors; monitoring how they feel; auditory feedback about progress; visual feedback about progress; and reminders to eat well. They were also asked how many of their current consumers could safely use a cell phone, as well as how many of their former consumers now in the community could safely use a cell phone.

\section{Results}

The clinicians surveyed reported that the majority (56.24\%) of their current consumers could safely use a cell phone. An even greater proportion (70.24\%) of community-based consumers were viewed as capable of safely using a cell phone. The following table indicates the percentage of consumers that clinicians believed could benefit from the functions offered by TherAPPist.
Table 1. Clinicians' estimates of how many of their current consumers would benefit from the following functions provided by the TherAPPist mobile application: Means and Standard Deviations (SD).

\begin{tabular}{|c|c|c|}
\hline Function & $\begin{array}{c}\text { Mean Endorsement } \\
\text { Percent }\end{array}$ & SD \\
\hline $\begin{array}{l}\text { Reminders about their } \\
\text { strengths }\end{array}$ & $73.24 \%$ & \pm 27.83 \\
\hline $\begin{array}{l}\text { Redirection when } \\
\text { upset }\end{array}$ & $72.95 \%$ & \pm 24.20 \\
\hline $\begin{array}{l}\text { Positive activities } \\
\text { when bored }\end{array}$ & $72.29 \%$ & \pm 22.37 \\
\hline $\begin{array}{l}\text { Listening to music } \\
\text { when upset }\end{array}$ & $71.07 \%$ & \pm 27.46 \\
\hline $\begin{array}{l}\text { Refocusing when } \\
\text { upset }\end{array}$ & $68.48 \%$ & \pm 29.25 \\
\hline $\begin{array}{l}\text { Reminders to take } \\
\text { medication }\end{array}$ & $67.86 \%$ & \pm 22.96 \\
\hline $\begin{array}{l}\text { Visual Feedback about } \\
\text { progress }\end{array}$ & $67.52 \%$ & \pm 27.15 \\
\hline $\begin{array}{l}\text { Visual Feedback about } \\
\text { progress }\end{array}$ & $66.42 \%$ & \pm 25.94 \\
\hline $\begin{array}{l}\text { Auditory Feedback } \\
\text { about progress }\end{array}$ & $64.29 \%$ & \pm 24.16 \\
\hline $\begin{array}{l}\text { Reminders about } \\
\text { supportive others }\end{array}$ & $62.76 \%$ & \pm 29.34 \\
\hline $\begin{array}{l}\text { Monitoring how they } \\
\text { feel }\end{array}$ & $58.83 \%$ & \pm 30.57 \\
\hline $\begin{array}{l}\text { Relaxation induction } \\
\text { when upset }\end{array}$ & $57.45 \%$ & \pm 28.81 \\
\hline $\begin{array}{l}\text { Monitoring their goal } \\
\text { behaviors }\end{array}$ & $53.10 \%$ & \pm 31.58 \\
\hline Reminders to eat well & $49.71 \%$ & \pm 23.16 \\
\hline Journaling & $40 \%$ & \pm 26.18 \\
\hline
\end{tabular}

When the most highly rated types of assistance (strengths reminders, redirection, positive activities, music) were compared, paired samples t-tests showed no significant differences in their perceived value to consumers. When the next tier of types of assistance (refocusing, medication reminders, visual progress feedback, generalization help, auditory progress feedback, supportive others, monitoring feelings, and relaxation induction) were compared, paired samples ttests indicated that relaxation induction was seen as less beneficial to consumers than many other interventions in the second tier: refocusing $(t=3.14$, $\mathrm{df}=41, \mathrm{p}<.01)$, medication reminders $(\mathrm{t}=3.46, \mathrm{df}=41$, 
$\mathrm{p}<.001)$, and generalization help $(\mathrm{t}=2.38, \mathrm{df}=41, \mathrm{p}<.05)$. The other helping components in this tier were rated as comparably important. Relaxation induction was also seen as likely to benefit fewer consumers than all of the four first tier interventions,

Among the three lowest rated interventions, journaling was perceived to benefit the fewest of these consumers. Paired samples tests showed that journaling was rated significantly lower than monitoring feelings $(t=5.75, d f=41, p<.001)$, monitoring behavior $(\mathrm{t}=4.30, \mathrm{df}=41, \mathrm{p}<.001)$, and eating reminders $(\mathrm{t}=2.87, \mathrm{df}=41, \mathrm{p}<.01)$.

\section{Discussion}

Clinicians reported that over two-thirds of their consumers in the community could safely make use of cell phone technology; a lower percentage, but still a majority, of those currently in inpatient settings were seen as candidates for this technology. A majority of consumers were seen as likely to benefit from the reminders about their strengths, redirection, positive activities, and access to music that the appwill provide. The assistance with refocusing, medication adherence, auditory and visual progress feedback, and generalization offered by therAPPist were also expected to benefit over two-thirds of these clients. Somewhat fewer consumers were seen as likely to benefit from the journaling, relaxation, and behavioral tracking features of the app. Since using these latter features may be seen as requiring greater cognitive skills, it may be especially important to simplify and abbreviate these features of the app to make them suitable for this group of consumers.

These findings suggest that additional research using this technology with such consumers would be valuable.This inexpensive app (99cents) offers individualized (via customizable components), timely (via immediacy of help), and responsive (via provision of feedback on progress) assistance. If effective in promoting greater self-control, this app has the potential to enhance consumers' lives and reduce healthcare costs. When fleeting emotional reactions are handled inappropriately, unsafe behaviors can necessitate expensive hospitalization or arrest. A typical inpatient facility stay costs $\$ 1227$ per day and lasts 6.6 days.

The psychological price of an involuntary commitment on a locked unit can be even more challenging to endure. Similarly, being arrested and secluded in jail imposes both financial and psychic costs. According to a June 2010 article published in The Economist, yearly spending on a single inmate ranges from $\$ 18,000$ in Mississippi to approximately $\$ 50,000$ in California, where the average bill per day to house an inmate in state prisons is about $\$ 129$. If this app can even modestly reduce episodes of incarceration among those diagnosed with schizophrenia, it will represent an important step forward.

\section{Future Work}

Often, a small intervention can make a big difference in preventing a maladaptive escalation, if it can be offered at just the right time. It is hoped that TherAPPist will enable consumers to have more control over delivery of services, making treatment more responsive to their immediate needs. TherAPPist reduces the need to wait for assistance, which may make all the difference for many easily frustrated, impulsive consumers.

Since executive function impairment underlies so many forms of personally and socially maladaptive behavior, mobile applications designed to supplement self control capabilities have the promise to enhance lives. As technology advances, and devices incorporate more biometric data, future therapeutic mobile applications will serve as tools to improve behavioral functioning, health, and subjective well-being.

The need to curb growth of healthcare spending mandates the development of more creative means of assisting those with various chronic illnesses. The timely, inexpensive, continuous support made possible by mobile applications such as TherAPPist could dramatically improve the lives of those with schizophrenia and other brain disorders, without unduly straining budgets.

\section{Disclosure}

The authors jointly developed and are disseminating one type of mobile application for enhancing the treatment of schizophrenia (TherAPPist). Although the survey used in this study assessed the general feasibility of mobile applications in helping these consumers and the potential utility of functions potentially offered via various generic mobile applications, a competing interest exists here due to the potential financial gain associated with the marketing of TherAPPist. 


\section{References}

[1] B. Bowens "A cognitive regulatory control model of schizophrenia" Brain Research Bulletin, 85, 2011, 36-41.

[2] K.T. Mueser, P.W. Corrigan, D.W. Hilton, B. Tanzman, A. Schaub, S. Gingerich, S.M. Essock, N. Tarrier, B. Morey, S. Vogel-Scibilia, M.I. Herz "Illness management and recovery for severe mental illness: a review of the research" Psychiatric Services, 53, 2002, 1272-1284.

[3] J.A. Lieberman, T.S. Stroup, J.P. McEvoy, M.S. Swartz, R.A. Rosenheck, D.O. Perkins, R.S. Keefe, S.M. Davis, C.E. Davis, B.D. Lebowitz, J. Severe, J.K. Hsiao "Effectiveness of antipsychotic drugs in patients with chronic schizophrenia" New England Journal of Medicine, 2005, 353, 1209-1223.

[4] P.J. Weiden, C. Kozma, A. Grogg, J. Locklear "Partial compliance and risk of rehospitalization among California Medicaid patients with schizophrenia" Psychiatric Services, 2004, 55(8), 886-91.

[5] R.E. Drake, M.R. Merrens, D.W. Lynde "EvidenceBased Mental Health Practice: A Textbook" 2005, New York, Norton.

[6] I. Hasson-Ohayon, D. Roe, S. Kravetz "A Randomized Controlled Trial of the Effectiveness of the Illness Management and Recovery Program" Psychiatric Services, 2007, 58, 1461-1466.

[7] M. Benton \& H.E. Schroeder "Social Skills Training With Schizophrenics: A Meta-Analytic Evaluation" Journal of Consulting and Clinical Psychology, 1990, 58(6).

[8] M.I. Herz, J.S. Lamberti, J. Mintz, R. Scott, S.P. O’Dell, L. McCartan \& G. Nix "A Program for Relapse Prevention in Schizophrenia" Archives of General Psychiatry, 2000, 57(3), 277-83.

[9] D. Shohamy, P. Mihalakos, R. Chin, B. Thomas, A.D. Wagner, \& C. Tamminga "Learning and generalization in Schizophrenia: Effects of disease and antipsychotic drug treatment" Biological Psychiatry, 2010, 67(10), 926-932.

[10] C.A. Chambliss “Group Involvement Training” 1988, New Harbinger Publications, Inc.

[11] R.A. Chapman, K.J. Shedlack \& J. France "StopThink-Relax: An adapted self-control training strategy for individuals with mental retardation and coexisting psychiatric illness" Cognitive and Behavioral Practice, 2006, 13, 205-214.

[12] A. Kopelowicz, R.P. Liberman, \& R. Zarate "Recent Advances in Social Skills Training for
Schizophrenia" Schizophrenia Bulletin, 2006, 32(Suppl 1), S12-S23.

[13] B. Vreeland, S. Minsky, P.T. Yanos, M. Menza, M. Gara, E. Kim, A.M. Toto, L. Allen "Efficacy of the Team Solutions program for educating patients about illness management and treatment" Psychiatric Services, 2006, 57, 822-828.

[14] A.F. Lehman \& D.M. Steinwachs "Patterns of usual care for schizophrenia: initial results from the Schizophrenia Patient Outcomes Research Team (PORT) client survey" Schizophrenia Bulletin, 1998 24, 11-20.

[15] M. Olfson, D. Mechanic, C.A. Boyer, S. Hansell, J. Walkup, \& P.J. Weiden "Assessing clinical predictions of early rehospitalization in schizophrenia" Journal of Nervous and Mental Disease, 1999, 187(12):721-9.

[16] H.R. Lamb "Structure: the neglected ingredient of community treatment" Archives of General Psychiatry, 1980, 37(11):1224-8.

[17] S. Gingerich, K.T. Mueser "Illness management and recovery, in Evidence-Based Mental Health Practice: A Textbook" Edited by R.E. Drake, M.R. Merrens, D.W. Lynde, 2005, New York, Norton.

[18] P.H. Lysaker, L.W. Davis, J. Lightfoot, N. Hunter, \& A. Stasburger "Association of neurocognition, anxiety, positive and negative symptoms with coping preference in schizophrenia spectrum disorders" Schizophrenia Research, 2005, 80(2-3):163-71. Epub 2005 Aug 24.

[19] G. Gay, J.P. Pollak, P. Adams, J.P. Leonard "Aurora. Pilot study of Aurora, a social, mobile phone-based emotion sharing and recording system" Journal of Diabetes Science and Technology, 2011; 5(2):325332.

[20] M.E. Morris, Q. Kathawala, T.K. Leen, E.E. Gorenstein, F. Guilak, M. Labhard, W. Deleeuw "Mobile Therapy: Case Study Evaluations of a Cell Phone Application for Emotional Self-Awareness" Journal of Medical Internet Research, 2010, 12(2): e10.

[21] V.L. Franklin, A. Greene, A. Waller, S.A. Greene, and C. Pagliari "Patients' Engagement with 'Sweet Talk' - a Text Messaging Support System for Young People with Diabetes" Journal of Medical Internet Research, 2008, 10(2), e20.

[22] A.G. Logan, W.J. McIsaac, A. Tisler, M.J. Irvine, A. Saunders, A. Dunai, C.A. Rizo, D.S. Feig, M. Hamill, M. Trudel, J.A. Cafazzo "Mobile Phone-based Remote Patient Monitoring System for 
Management of Hypertension in Diabetic Patients" American Journal of Hypertension, 2007, 20 (9), 942-948.

[23] D. Ryan, W. Cobern, J. Wheeler, D. Price, \& L. Tarassenko "Mobile Phone Technology in the Management of Asthma" Journal of Telemedicine and Telecare, 2005, 11 (Suppl 1): 43-46.

[24] G. Lee "PmEB: A Mobile Phone Application for Monitoring Caloric Balance" CHI '06 Extended Abstracts on Human Factors in Computing Systems. 22-27 Apr. 2006, 1013-1018, ACM.

[25] A. Smith "Americans and Their Cell Phones" A Pew Internet \& American Life Project, 2011.

[26] Wikimedia, "Wikimedia Traffic Analysis Report Operating Systems", Aug. 2012, [Online] http://stats.wikimedia.org/archive/squid_reports/ 2012-08/SquidReportOperatingSystems.htm.

[Accessed 15 Oct. 2012]

TherAPPist links

Link to Android version:

play.google.com/store/apps/details?id=com.therappist. therappist

Link to Website:

http://asquaredapplications.com/TherAPPist.html

Link to iOS version:

https://itunes.apple.com/us/app/therappist/id63

3327098? $\mathrm{ls}=1 \& \mathrm{mt}=8$ 